\title{
Community Asset Mapping
}

Community asset mapping is the process through which local leaders identify and take inventory of physical and social assets in the city spanning from major industries to cultural groups, and community leaders. Asset mapping is a community planning tool intended for local municipalities and community leaders to gain new perspectives and knowledge of existing community assets. Community assets contribute to an enhanced quality of life and makeup both the physical and social environment of any city. Community assets can range from the city's natural environment, physical structures, social and cultural values and knowledge, public services, and jobs and business. In identifying the value already present in one's community, a strategic planning process can proceed to activate new and underutilized assets. To create the conditions for investment and belonging amongst a diverse population, a wide range of stakeholders must collaborate in this process.

An infrastructure of support can be built into existing physical community assets or leveraged by identifiable community stakeholders to foster inclusion. An infrastructure of support in this context refers to the ability of city leaders to adapt existing industries like a local hospital, school or library, and also train front-line workers and employers to confront issues of diversity and foster inclusion. To attract and retain newcomers, such adaptations to businesses may come in the form of inclusive hiring policies, the offering of language classes, or providing instruction and policy in multiple languages.

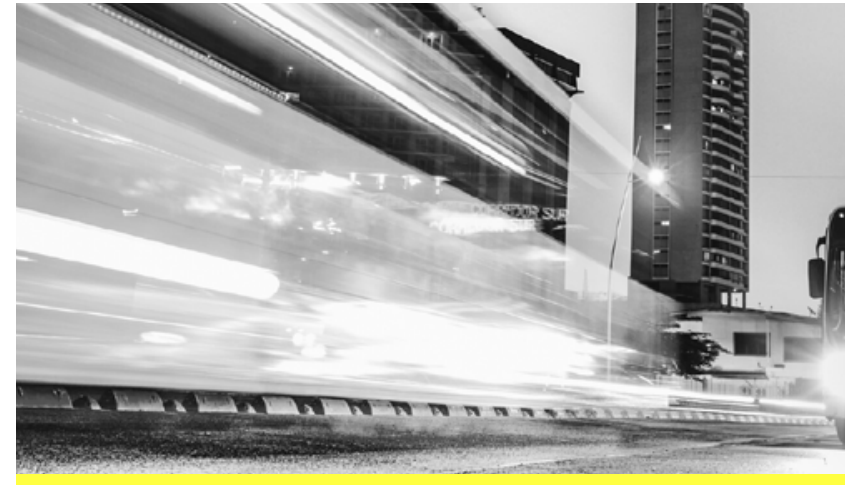

\section{Potential list of stakeholders, businesses, sectors, and local organizations}

> Municipal Economic Development

> Local/Regional Economic Development agencies

$>$ Municipal newcomer office

$>$ Chamber of Commerce

> Small Business Enterprise Centres

> Downtown Improvement Associations

$>$ Employment $\&$ Education Centres

$>$ Local Employers

> District School Board - International Education

> Community colleges - International Education

> Universities - International Education

> The credit union, or local financial institution

> Immigrant serving organizations

> Anchor institutions, for example hospitals, regional health centers, public libraries, public art gallery/museums,

> Tourism/city branding: arts $\&$ culture; sport/ 